\title{
Actitudes hacia la Violencia Contra la Mujer en estudiantes de cuarto año de secundaria de Lima Metropolitana
}

\author{
Attitudes towards Violence Against Women in fourth grade students of \\ Lima Metropolitana
}

\section{Daniel Efraín Olarte Calvera ${ }^{1}$}

\section{Revista \\ Educación y Sociedad}

Citar como: Olarte, D. (2020). Actitudes hacia la Violencia Contra la Mujer en estudiantes de cuarto año de secundaria de Lima Metropolitana. Revista Educación y Sociedad, 01(02), 2-18. doi: 10.53940/reys.v1i2.55

Artículo recibido: 10-06-2020 Artículo aprobado: 19-10-2020 Arbitrado por pares

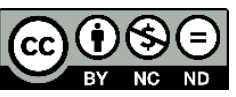

\section{ACEES}

\section{Resumen}

La presente investigación plantea el estudio de las actitudes hacia la violencia contra la mujer en estudiantes de cuarto año de secundaria de instituciones educativas estatales y no estatales. Se compara las actitudes hacia la violencia contra la mujer según el factor género y considerando el tipo de gestión educativa. En la investigación participaron 196 alumnos del cuarto año de secundaria provenientes de colegios estatales y no estatales de colegios de las UGEL 2 y 3 de Lima Metropolitana. El diseño que se empleó en esta investigación fue de tipo descriptivo - comparativo. Los resultados muestran que los estudiantes hombres de cuarto año de secundaria presentan, en su mayoría, actitudes favorables hacia la violencia contra la mujer, a diferencia de las mujeres que presentan, en su mayoría, una actitud de indiferencia hacia la violencia contra la mujer. Asimismo, se muestra que los estudiantes de cuarto año de secundaria pertenecientes a una institución educativa estatal presentan, en su mayoría, actitudes favorables hacia la violencia contra la mujer, a diferencia de los estudiantes pertenecientes a una institución educativa no estatal.

Palabras clave: violencia contra la mujer, estudiantes de cuarto año de secundaria, instituciones educativas estatales y no estatales

Abstract

This research proposes the study of attitudes towards violence against women on fourth grade secondary students from public and private educational institutions of Metropolitan Lima. It compares attitudes towards violence against women according to the gender factor and considering the school type of management. The research involved 196 fourth grade secondary students from state and non-state schools from UGEL 02 and 03 schools in Metropolitan Lima. The design used in this research was descriptive-comparative. The results show that the male fourth grade secondary students present, in their majority, favorable attitudes towards violence against women, unlike the women who present, in their majority, an attitude of indifference towards violence against women. Also, the fourth grade secondary students belonging to a public institution present, in their majority, favorable attitudes towards violence against women, unlike the fourth grade secondary students belonging to a private institution.

Key words: violence against women, fourth grade secondary students, public institution, private institution 


\section{Introducción}

Desde los inicios de la formación de la sociedad la violencia ha sido un medio primitivo de persuasión que utilizaban algunos individuos o grupos de personas para someter a los demás, con la finalidad de ubicarse en un nivel jerárquico superior, lo cual implicaba múltiples beneficios para quienes dominaban, principalmente el de supervivencia y prevalencia. Esta violencia se mantiene en nuestra contemporaneidad como uno de los síntomas más relevantes, acrecentándose con el pasar del tiempo (Ons, 2009).

Esta violencia se ha manifestado y dirigido con mayor fuerza hacia los grupos sociales que no se les ha permitido empoderarse totalmente, debido a un sistema social rígido y discriminatorio. Entre estos grupos, se encuentra el caso particular de las mujeres, maltratadas por el sistema político de su país, por sus superiores en sus trabajos y por la sociedad en general.

Actualmente la violencia contra la mujer, o violencia de género, es considerada como las acciones o comportamientos basados en su género que causen muerte, daño o sufrimiento físico, sexual o psicológico a la mujer, tanto en el ámbito público como en el privado (OEA, como se cita en Velázquez, 2003).

Según la Organización Panamericana de la Salud (Solano y Velzeboer, 2003), los tipos de violencia son: a) violencia física: causa daño no accidental a otra persona mediante el uso de la fuerza física o algún tipo de arma, b) violencia psicológica: toda conducta que ocasiona daño emocional, disminuye la autoestima, perjudica o perturba el sano desarrollo de la mujer u otro integrante de la familia, c) violencia sexual: la Organización Mundial de la Salud (2002) lo define como todo acto sexual o la tentativa de consumar un acto sexual no deseado, las insinuaciones sexuales no deseadas, las acciones para comercializar o utilizar de cualquier otro modo la sexualidad de una persona mediante la coacción por otra persona independientemente de la relación de ésta con la víctima y, en todo ámbito, incluyendo el hogar y el lugar de trabajo, d) violencia patrimonial o económica: Se produce cuando las acciones u omisiones del agresor afectan la vida y/o la supervivencia económica de miembros de la familia.

Según Ortega (2011), cuando se hace referencia a la violencia contra la mujer se distinguen tres niveles: a) violencia conyugal, hace referencia a la violencia que un cónyuge o ex-cónyuge, novio o ex-novio, pareja o ex-pareja, ejerce sobre su mujer, b) violencia contra la mujer (o de género), se refiere a la violencia que una mujer sufre en la sociedad por su condición de mujer y es ejercida por instituciones, c) violencia doméstica, hace referencia a todo sistema de valores complementarios negativos, dentro del que lo femenino siempre es interpretado como sumisión y subordinación frente a lo masculino, que es interpretado como dominio.

La OMS, en su "Informe Mundial de Violencia y Salud" (2002), señala las siguientes consecuencias psicológicas como los más frecuentes: Abuso de tabaco, alcohol y otras drogas, depresión, ansiedad, trastornos alimentarios, trastornos del sueño, sentimientos de vergüenza y culpa, fobias y trastorno de pánico, inactividad física, baja autoestima, trastorno de estrés postraumático, trastornos psicosomáticos, problemas en la conducta sexual y disfunciones sexuales.

En respuesta a esta realidad, las instituciones internacionales, los estados y la sociedad en general han tratado de regular el ejercicio arbitrario de la violencia, con el objetivo de proteger a los más vulnerables, en principio con la Declaración Universal de los Derechos Humanos en 1948, afirmando la igualdad entre hombres y mujeres. Posteriormente, con la Convención para la Eliminación de todas las formas de Discriminación contra la Mujer en 1974, la Declaración sobre la Erradicación de la Violencia contra las Mujeres en 1993 y, por parte de la Organización Mundial de la Salud (OMS), la declaración a la violencia, incluyendo la violencia contra la mujer y los niños, como una prioridad de salud pública que requería acción urgente (Güezmes, Palomino y Ramos, 2002). 
A pesar de todo el esfuerzo, en el mundo aún se evidencian estadísticas preocupantes, como las cifras del "Informe sobre Población Mundial 2000" de Naciones Unidas y el “Informe Mundial sobre Violencia y Salud" de la OMS, donde se reporta que una de cada tres mujeres en el mundo ha padecido o padece malos tratos o abusos (Krug, Dahlberg, Mercy, Zwi, Lozano, como se cita en Lorente, 2008). En ese sentido, Ruiz (2006) afirma que esta violencia desatada hacia las mujeres es la prueba de que se necesitan más que leyes para combatir está lógica patriarcal.

Ortega (2011) plantea que se debe abordar la problemática desde una perspectiva nueva que permita incidir en las causas de esta violencia, considerando los valores culturales que hacen que este tipo de violencia prevalezca y se extienda por todo el mundo.

Teubal (2001) considera que la violencia se genera por la confluencia de múltiples factores interrelacionados a partir de una sociedad constituida y formada por desigualdades que, a su vez, son mantenidas por un sistema de representaciones sociales que otorgan diferentes niveles de jerarquía y poder a los miembros de una familia, sea hombre, mujer, niño o niña. Es el sistema de valores actual el que contribuye a que un género determinado esté siempre en por encima del otro, imponiendo su supuesta superioridad y que, por lo general, en las culturas sea el género masculino quien se imponga.

Respecto a la teoría del género, algunos autores (Cazes, 2000, Fernández, 1998, Lamas, 2003, como se cita en Rocha, 2011) sugieren que ser hombre o ser mujer resulta de una asignación arbitraria y no por una situación natural, por lo que es necesario distinguir entre las diferencias que son inherentes y las que son aprendidas. Por ello, se considera que ser hombre o mujer es resultado de varios procesos: psicológicos, sociales y culturales, a partir de los cuales se asigna un significado para cada uno. En ese sentido, para cada cultura existe una noción específica de lo que significa ser hombre o ser mujer. Estos significados están conformados por características, valores, comportamientos, reglas, prohibiciones y expectativas que son transferidos y reforzados en los individuos como parte de su proceso de identidad.

El término "género" engloba los diversos rasgos y características que son socioculturalmente apropiados para hombres y mujeres, por lo cual, fundamentalmente, el género hace referencia a categorías sociales (Unger, 1979, como se cita en Matud et al., 2002).

El rol de género puede definirse como aquellas expectativas y conductas que se asocian a ser hombre o ser mujer. Se espera que los hombres sean fuertes, competitivos e independientes y que las mujeres sean cariñosas, expresivas emocionalmente y serviciales. Es decir, se espera que las mujeres sean femeninas y los hombres masculinos. (Hegelson, 2002, como se cita en Matud et al., 2002).

Identidad de género se refiere al fenómeno, que tiene lugar generalmente en la infancia, por el que los individuos se perciben a sí mismos como hombres o como mujeres (Winstead y Derlega, 1993, como se cita en Matud et al., 2002). En el caso de la identidad de género, se pueden encontrar afirmaciones específicas que integran la cultura de género conformados por los estereotipos de género. Estas son ideas preconcebidas relativas al significado de ser varón o mujer, que se expresan en características y conductas valoradas socialmente entre los individuos en función al sexo biológico (Rocha, 2011).

Las relaciones de género se van construyendo en diversos ámbitos, como el doméstico, social y laboral, donde se le otorga al varón mayor importancia sobre la mujer, dándole poder real y simbólico, generándose aquella desigualdad e inequidad (Ministerio de Salud, 2006, Ministerio de la Mujer y Desarrollo Social, 2008, Macazana, 2010). Entonces, la violencia es inseparable de la noción de género porque se basa y se ejerce en y por la diferencia social y subjetiva entre los sexos (Velázquez, 2003).

Para el abordaje de la violencia contra la mujer se necesita de un modelo que integre el análisis de los diversos ámbitos involucrados y sus interrelaciones: a nivel del individuo, las relaciones de pareja y de familia, la vida comunitaria y social, el contexto socioeconómico y las políticas de educación y empleo, 
etc. (Comisión Económica para América Latina y el Caribe [CEPAL], 2007) Por ello, se considera mejor plantear esta problemática basándose en la teoría de Bröfenbrener sobre los sistemas ecológicos.

Horno (2009) refiere que el marco socio-ecológico proporciona un instrumento de análisis social para cualquier problema o dinámica social en la que se quiera intervenir. Este autor hace referencia a dicha teoría explicándola de la siguiente manera: Una persona se desarrolla en una serie de entornos que se engloban los unos a los otros. En cada nivel entra en juego una multiplicidad de factores que se interrelacionan entre sí. El primer nivel es la persona en sí misma, con sus características de personalidad, temperamentales, su historia personal y otros factores. El segundo nivel, o microsistema, engloba las relaciones interpersonales que la persona establece con su familia, sus amigos, en la escuela, es decir, todos los entornos en los que la persona desarrolla relaciones personales. El tercer nivel o macrosistema hace referencia al entorno donde estas relaciones tienen lugar: El barrio, la comunidad, las instituciones que trabajan en esa comunidad, etc., se refiere a todo el entorno social. El cuarto y último círculo, Ilamado en el modelo exosistema, engloba los factores de la cultura en la que se enmarca este entorno social, factores como los valores culturales, la religión o los medios de comunicación.

Carmona, Deporto, Corral, Villalobos y López (2005), en Cuba, investigaron respecto a la incidencia y prevalencia de la violencia contra la mujer tanto física como psicológica y sexual ejercida por su pareja dentro del ámbito universitario. La muestra total fue de 538 mujeres estudiantes de la Universidad Autónoma de Ciudad Juárez, entre 17 y 50 años de edad y con pareja; se tuvo en cuenta el nivel curricular y socioeconómico, la religión y tipo y antigüedad en la relación. Se utilizó un inventario de violencia con 35 reactivos estructurado como una escala Likert, referente a las expresiones de violencia física, sexual y psicológica. En cuanto a los resultados, se obtuvo que el $94 \%$ de las mujeres habían sufrido por lo menos alguna vez violencia, en sus diferentes tipos, dentro de sus relaciones de pareja. Además, 4 de cada 10 mujeres habían sufrido violencia física por lo menos alguna vez, 4 de cada 10 mujeres habían vivido maltrato sexual por lo menos alguna vez y 9 de cada 10 mujeres habían vivido violencia psicológica por lo menos alguna vez.

Rodríguez, Sánchez y Alonso (2006), en España, investigaron las creencias de adolescentes y jóvenes en torno a la violencia de género y las relaciones de pareja. La muestra del estudio estuvo integrada por 152 jóvenes, siendo 76 estudiantes universitarios en su primer año de carrera y que cursaban estudios de Trabajo Social y Ciencias Empresariales en el Centro de Estudios Universitarios de Talavera de la Reina Universidad de Castilla La Mancha. Los 76 jóvenes restantes eran estudiantes de cuarto año de la ESO (Enseñanza Secundaria Obligatoria) en el Instituto de Educación Secundaria San Isidro, localizado en Talavera de la Reina. De todos ellos, 105 eran mujeres, 47 hombres y sus edades oscilaban entre 15 y 23 años, siendo la edad media de 17,9 años. El instrumento utilizado estaba conformado por 23 preguntas que recogían información de diferentes variables. En cuanto a los resultados, se halló que el $70 \%$ de estos estudiantes reconocían que hombre y mujer asumían posiciones y funciones diferentes dentro de la relación, atribuyendo el rol protector al hombre y quedando la mujer supeditada a su cuidado y atención, ya que implícitamente es percibida como el miembro más vulnerable de la pareja. El $25 \%$ se mostró totalmente de acuerdo con la afirmación de que el "Hombre ha de triunfar laboral y socialmente". Por tanto, se confirmaba los rasgos estereotipados atribuidos a hombre y mujer, destacando claramente el ámbito laboral, social y de triunfo masculino. Por último, el $12 \%$ de los estudiantes mostraba su acuerdo total a la afirmación de que "El maltrato a la mujer es un problema de la pareja, de nadie más", evidenciando que aún hoy hay personas tan jóvenes con este tipo de pensamiento, en cuanto que conlleva la explicación de la violencia basada en características de las personas, no en que sea un problema social que requiera ser solucionado por todos.

Ferrer, Bosch, Ramis y Navarro (2006), en España, realizaron una investigación respecto a las creencias y actitudes sobre la violencia contra las mujeres en la pareja: Determinantes sociodemográficos, familiares y formativos. Se realizó en una muestra de 1395 estudiantes universitarios, 470 varones y 898 mujeres; el resto no indicó su género. Se utilizó el Inventario de Pensamientos Distorsionados sobre la Mujer y la Violencia. Los resultados obtenidos indicaban que los factores estudiados explicaban un 
pequeño porcentaje de estas creencias y actitudes (entre el $5 \%$ y el 13\% según los casos) y se destacaba el hecho de que la variable explicativa más importante en todos los casos era el género. Además, los resultados sugirieron que la experiencia vital de los estudiantes que formaban parte de la muestra parecía desempeñar un papel en el sexismo y en las actitudes y creencias hacia la violencia contra las mujeres en la pareja. El tipo de educación recibida y los modelos observados contribuyeron también a predecir el sexismo y la tolerancia hacia la violencia contra las mujeres en la pareja.

Gracia, Herrero, Lila y Fuente (2010) investigaron las actitudes hacia la violencia de pareja contra la mujer en inmigrantes latinoamericanos situados en España. La muestra estuvo conformada por los datos que incluyó una encuesta nacional española, con una muestra de 2498 adultos (18 o más años) realizada en 2004 por el Centro de Investigaciones Sociológicas, para abordar diferentes cuestiones sobre la violencia doméstica. Además, participaron en el estudio 399 inmigrantes latinoamericanos que en el momento de la investigación residían en dos comunidades autónomas en España: Asturias y Valencia. Los resultados mostraron diferencias significativas entre españoles e inmigrantes en las percepciones y actitudes hacia la violencia de pareja contra la mujer. Los análisis también mostraron que las actitudes positivas hacia la denuncia, manifestadas por los inmigrantes latinoamericanos, fueron más frecuentes entre aquellos que eran menos tolerantes con la violencia de pareja contra la mujer, la percibían como un problema importante en la sociedad y no tendían a culpar a las víctimas.

Anicama, Vizcardo, Carrasco y Mayorga (1999) realizaron un estudio epidemiológico sobre la violencia y comportamientos asociados en Lima Metropolitana y Callao, donde evaluaron 3590 hogares debidamente muestreados y utilizando un instrumento especialmente construido para el caso. Los resultados fueron obtenidos por áreas: en el área familiar, se halló que el 35.4\% recibieron violencia psicológica por parte de su pareja, el $17.4 \%$ señaló haber sido víctima de violencia física por parte de su pareja. En cuanto al maltrato infantil, el $36.2 \%$ de los padres señalaron maltratar psicológicamente a sus hijos. En el área de comportamientos asociados, se encontró que las principales sustancias psicoactivas que alguna vez fueron consumidas regularmente por los encuestados fueron: Alcohol $27.5 \%$, tabaco $18.7 \%$ y medicamentos no recetados en un $3.7 \%$.

Chuquimajo (2000) realizó una investigación respecto a las actitudes hacia la violencia contra la mujer en la relación de pareja en estudiantes de quinto año de secundaria de Lima Metropolitana. La muestra estuvo conformada por 400 sujetos, 219 varones y 181 mujeres, a quienes se les aplicó la Escala de Actitudes hacia la Violencia contra la Mujer en la Relación de Pareja V - M, elaborada por la investigadora. Los resultados más importantes que se obtuvieron de dicha investigación fueron los siguientes: Los estudiantes varones del quinto año de secundaria de instituciones educativas estatales presentaron actitudes más favorables hacia la violencia contra las mujeres en la relación de pareja que las mujeres del mismo año. Además, los estudiantes de quinto año de secundaria de instituciones educativas estatales que presenciaron peleas y discusiones entre sus padres, mostraron actitudes de mayor rechazo hacia la subescala de violencia como medio; asimismo, los estudiantes de quinto año de secundaria de instituciones educativas estatales que presenciaron peleas o discusiones casi todos los días, por lo menos una vez a la semana y una vez al año, presentan actitudes homogéneas.

Elizalde (2001) Ilevó a cabo un estudio sobre las actitudes hacia la violencia y rasgos de personalidad en estudiantes universitarios de Lima. La muestra se compuso de 277 estudiantes, 133 varones y 144 mujeres, que cursaban entre el primer y quinto año de la carrera profesional en el año 1998. Para dicha investigación empleó las fichas sociodemográficas y la Escala de Actitudes Hacia la Violencia (AHV), siendo esta de tipo Likert y de 60 ítems; asimismo utilizó el Cuestionario de Análisis Clínico de Krug (CAQ II) de 144 ítems. Entre los resultados más importantes de la investigación, se encontró que los varones mostraban mayores actitudes favorables hacia la violencia que las mujeres. Halló diferencia estadísticamente significativa entre las actitudes favorables hacia la violencia en el área de relación de pareja y relación familiar en estudiantes que trabajaban, siendo probable el estrés laboral un factor causal. 
Macazana (2010) realizó una investigación respecto a las actitudes hacia la violencia contra la mujer en la relación de pareja en estudiantes universitarios de cuatro universidades de Lima Metropolitana, considerando el género, nivel socioeconómico y que hayan tenido o no una pareja. La muestra estuvo constituida por 467 sujetos del tercer año de estudios universitarios, tanto de gestión estatal como particular, 170 varones y 297 mujeres. Se elaboró el instrumento denominado Escala de Actitudes V-M (CM). En base a los resultados encontrados, se afirmó que existían actitudes favorables hacia la violencia contra la mujer en la relación de pareja por parte de los hombres y que era distinto en cuanto a las mujeres, pues mostraban actitudes de indiferencia.

Es este proceso de la violencia en la sociedad, principalmente hacia la mujer, el que motiva a indagar sobre la situación de este fenómeno en nuestra época y, también, la percepción de los adolescentes sobre este tema. Asimismo, se busca dilucidar el rumbo de este fenómeno en particular.

De lo planteado, surgen las siguientes interrogantes: ¿Cuál es la actitud de los estudiantes de cuarto año de secundaria de las UGEL 2 y 3 de Lima Metropolitana hacia la violencia contra la mujer? ¿Difieren las actitudes hacia la violencia contra la mujer de los estudiantes de cuarto año de secundaria de las UGEL 2 y 3 de Lima Metropolitana en función a las variables género y tipo de gestión educativa?

Esta investigación se plantea los siguientes objetivos: a) describir las actitudes hacia la violencia contra la mujer de los estudiantes de cuarto año de secundaria de instituciones educativas estatales y no estatales de Lima Metropolitana, b) comparar las actitudes hacia la violencia contra la mujer entre los estudiantes varones y mujeres de cuarto año de secundaria de centros educativos de Lima Metropolitana y c) comparar las actitudes hacia la violencia contra la mujer entre los estudiantes de cuarto año de secundaria de instituciones educativas estatales y no estatales de Lima Metropolitana.

\section{Método}

\section{Diseño de Investigación}

El diseño que se empleó en esta investigación es de tipo descriptivo-comparativo. La información recolectada de los estudiantes de cuarto año de secundaria, sobre sus actitudes frente al fenómeno, se registrará bajo el mismo.

\section{Universo y muestra de investigación}

El universo de investigación estuvo conformado por los alumnos matriculados en cuarto año de secundaria de Lima Metropolitana. Se utilizó una muestra de 196 alumnos. Para la obtención de la muestra, se aplicó un procedimiento de muestreo probabilístico bi-etápico; en la primera etapa, en cada una de las UGEL, se eligió de manera aleatoria los centros educativos a ser incluidos en el estudio y, posteriormente, en cada colegio se eligieron de forma aleatoria los participantes que fueron evaluados.

\section{Instrumentos de investigación}

Para la presente investigación se utilizó la Escala de Actitudes hacia la Violencia contra la Mujer en la Relación de Pareja (Escala de Actitud V - M (CM)), adaptada para estudiantes de cuarto año de secundaria. Es una escala aditiva que corresponde a un nivel de medición ordinal. Está conformada por un conjunto de proposiciones que contiene juicios o reacciones que presentan los sujetos frente a este evento. Los contenidos incluyen los diversos tipos de violencia: Física, psicológica, sexual y económica. Las opciones de cada ítem varían en términos de grados y bajo la forma de alternativas: Muy de Acuerdo = MA; De Acuerdo $=$ A; Desacuerdo $=$ D; Muy en Desacuerdo $=$ MD. 


\section{Resultados}

Siguiendo las recomendaciones de Fernández y Fernández (2009) y Grisson y Kim (2012), se aplicó la prueba $U$ de Mann Whitney para contraste de la hipótesis y el estadístico dcliff para medir el tamaño del efecto.

En la comparación de las actitudes hacia la violencia contra la mujer, entre el grupo de hombres y mujeres (tabla 1), se aprecia que el primer grupo obtiene una media de rangos de 123.26 y el segundo grupo 84.13 , con un valor $U=2681.50$ y $Z=-4,657$ ( $p<.001$ ); ello permite señalar que existen diferencias estadísticamente significativas entre ambos grupos. Además, se observa que el coeficiente dcliff es de 0.40 , correspondiente a un efecto pequeño. De esta manera, se confirma la hipótesis general 1, siendo los hombres los que muestran una actitud más favorable hacia la violencia contra la mujer.

Tabla 1

Comparación de las actitudes hacia la violencia contra la mujer considerando la variable género

\begin{tabular}{ccccccc}
\hline Sexo & $\mathrm{n}$ & $\begin{array}{c}\text { Media de } \\
\text { Rangos }\end{array}$ & $\begin{array}{c}\text { Suma de } \\
\text { Rangos }\end{array}$ & $\begin{array}{c}\text { U de Mann } \\
\text { Whitney }\end{array}$ & $\mathrm{Z}$ & $\mathrm{d}_{\text {cliff }}$ \\
\hline Hombres & 72 & 123.26 & 8874.50 & & & \\
Mujeres & 124 & 84.13 & 10431.50 & & & \\
\hline Total & 196 & & & & & \\
\hline$* * * \mathrm{p}<.001$ & & & & & & \\
\hline
\end{tabular}

En la comparación de las actitudes hacia la violencia contra la mujer, entre los estudiantes que provienen de instituciones educativas estatales y no estatales (tabla 2), los resultados muestran que el primer grupo obtiene una media de rangos de 122.70 y el segundo 69.41 , con un valor $U=2172.500$ y $Z=-$ 6.549 ( $p$ < .001), indicando la existencia de diferencias estadísticamente significativas entre ambos grupos; además, el coeficiente dcliff alcanza un valor de 0.54 que se puede clasificar como un efecto mediano. De esta manera se confirma la hipótesis general 2, siendo los estudiantes de instituciones educativas estatales los que muestran una actitud más favorable hacia la violencia contra la mujer en la relación de pareja, a diferencia de los estudiantes de instituciones no estatales. 
Tabla 2

Comparación de las actitudes hacia la violencia contra la mujer considerando el tipo de institución educativa

\begin{tabular}{ccccccc}
\hline $\begin{array}{c}\text { Tipo de Institución } \\
\text { educativa }\end{array}$ & $\mathrm{n}$ & $\begin{array}{c}\text { Media de } \\
\text { Rangos }\end{array}$ & $\begin{array}{c}\text { Suma de } \\
\text { Rangos }\end{array}$ & $\begin{array}{c}\text { U de Mann } \\
\text { Whitney }\end{array}$ & $\mathrm{Z}$ & $\mathrm{d}_{\text {cliff }}$ \\
\hline Estatal & 107 & 122.70 & 13128.50 & & & \\
No Estatal & 89 & 69.41 & 6177.50 & & & \\
\hline Total & 196 & & & & & \\
\hline$* * * \mathrm{p}<.001$ & & & & & & \\
\hline
\end{tabular}

Se aprecia en la tabla 3, correspondiente a la comparación de medias de rango de las actitudes hacia la violencia contra la mujer en relación de pareja entre el grupo de hombres y mujeres, en función al factor la violencia como medio, que el primer grupo obtiene una media de rangos de 104.53 y el segundo grupo un 95.00 , con un valor $U=4029.50$ y $Z=-1.145$ ( $p>.05$ ), lo cual indica que no existen diferencias estadísticamente significativas entre ambos grupos; además, el coeficiente dcliff alcanza un valor de 0.10, que se puede clasificar como un efecto mínimo. Así, se puede señalar que no se valida la hipótesis específica H1.1, es decir, ambos grupos presentan actitudes similares en función al factor violencia como medio.

\section{Tabla 3}

Comparación de las actitudes hacia la violencia contra la mujer considerando la variable género en función al factor la violencia como medio

\begin{tabular}{ccccccc}
\hline Sexo & $\mathrm{N}$ & $\begin{array}{c}\text { Media de } \\
\text { Rangos }\end{array}$ & $\begin{array}{c}\text { Suma de } \\
\text { Rangos }\end{array}$ & $\begin{array}{c}\text { U de Mann } \\
\text { Whitney }\end{array}$ & $\mathrm{Z}$ & $\mathrm{d}_{\text {cliff }}$ \\
\hline Hombres & 72 & 104.53 & 7526.50 & & & \\
Mujeres & 124 & 95.00 & 11779.50 & & & \\
\hline Total & 196 & & & & & \\
\hline
\end{tabular}

En la tabla 4, correspondiente a la comparación de medias de rango de las actitudes hacia la violencia contra la mujer en relación de pareja entre hombres y mujeres en función al factor negación a la libertad, se observa que el primer grupo obtiene una media de rangos de 127.25 y el segundo grupo un 81.81 , con un valor $U=2394.00$ y $Z=-5.424$ ( $p<.001)$, indicando la existencia de diferencias estadísticamente significativas entre ambos grupos. El tamaño del efecto estimado a través del coeficiente dcliff fue de 0.99, que corresponde a un efecto grande. Confirmándose, así, la hipótesis específica H1.2: los varones muestran una actitud más favorable hacia la violencia contra la mujer en el factor negación a la libertad. 
Tabla 4

Comparación de las actitudes hacia la violencia contra la mujer considerando la variable género en función al factor negación a la libertad

\begin{tabular}{ccccccc}
\hline Sexo & $\mathrm{n}$ & $\begin{array}{c}\text { Media de } \\
\text { Rangos }\end{array}$ & $\begin{array}{c}\text { Suma de } \\
\text { Rangos }\end{array}$ & $\begin{array}{c}\text { U de Mann } \\
\text { Whitney }\end{array}$ & $\mathrm{Z}$ & $\mathrm{d}_{\text {cliff }}$ \\
Hombres & 72 & 127.25 & 9162.00 & & & \\
Mujeres & 124 & 81.81 & 10144.00 & & & \\
\hline Total & 196 & & & & & \\
\hline$* * * \mathrm{p}<.001$ & & & & & & \\
\hline
\end{tabular}

En la tabla 5, correspondiente a la comparación de medias de rango de las actitudes hacia la violencia contra la mujer en relación de pareja entre el grupo de hombres y mujeres en función al factor imposición de autoridad, se observa que los varones obtienen una media de rangos de 113.87 y las mujeres 89.58 , con un valor $U=3357.50$ y $Z=-2.909(p<.05)$, lo cual permite señalar que existen diferencias estadísticamente significativas entre ambos grupos. Además, el coeficiente dcliff alcanza un valor de 0.25 que se puede clasificar como un efecto pequeño. Confirmándose, de esta manera, la hipótesis específica H1.3: los hombres muestran una actitud más favorable hacia la violencia contra la mujer en el factor imposición de autoridad.

Tabla 5

Comparación de las actitudes hacia la violencia contra la mujer considerando la variable género, en función al factor imposición de autoridad

\begin{tabular}{ccccccc}
\hline Sexo & $\mathrm{n}$ & $\begin{array}{c}\text { Media de } \\
\text { Rangos }\end{array}$ & $\begin{array}{c}\text { Suma de } \\
\text { Rangos }\end{array}$ & $\begin{array}{c}\text { U de Mann } \\
\text { Whitney }\end{array}$ & $\mathrm{Z}$ & $\mathrm{d}_{\text {cliff }}$ \\
\hline Hombres & 72 & 113.87 & 8198.50 & & & \\
Mujeres & 124 & 89.58 & 11107.50 & & & \\
\hline Total & 196 & & & & & \\
\hline${ }^{*} \mathrm{p}<.05$ & & & & & & \\
\hline
\end{tabular}

En la comparación de medias de rango de las actitudes hacia la violencia contra la mujer en relación de pareja entre el grupo de hombres y mujeres en función al factor machismo (tabla 6), se observa que el primer grupo obtiene una media de rangos de 120.51 y el segundo grupo un 85.72 , con un valor $\mathrm{U}=2879.50$ y $Z=-4.154(p<.001)$, lo cual indica la existencia de diferencias estadísticamente significativas entre ambos grupos. El coeficiente dcliff alcanza un valor de 0.36, correspondiente a un efecto pequeño. Así, confirmándose la hipótesis específica $\mathrm{H}$ 1.4: son los varones quienes presentan una actitud más favorable hacia la violencia contra la mujer en el factor machismo. 
Tabla 6

Comparación de las actitudes hacia la violencia contra la mujer considerando la variable género, en función al factor machismo

\begin{tabular}{ccccccc}
\hline Sexo & $\mathrm{n}$ & $\begin{array}{c}\text { Media de } \\
\text { Rangos }\end{array}$ & $\begin{array}{c}\text { Suma de } \\
\text { Rangos }\end{array}$ & $\begin{array}{c}\text { U de Mann } \\
\text { Whitney }\end{array}$ & $\mathrm{Z}$ & $\mathrm{d}_{\text {cliff }}$ \\
\hline Hombres & 72 & 120.51 & 8676.50 & & & \\
Mujeres & 124 & 85.72 & 10629.50 & & $-4.154^{* * *}$ & 0.36 \\
\hline Total & 196 & & & & & \\
\hline$* * * \mathrm{p}<.001$ & & & & &
\end{tabular}

En el caso de la tabla 7, correspondiente a la comparación de medias de rango de las actitudes hacia la violencia contra la mujer en relación de pareja entre el grupo de hombres y mujeres en función al factor desconsideración, se observa que el primer grupo obtiene una media de rangos de 116.13 y el segundo grupo 88.26, con un valor $U=3194.50$ y $Z=-3.338$ ( $p<.001$ ), lo cual permite señalar que existen diferencias estadísticamente significativas entre ambos grupos. Observándose, también, que el coeficiente dcliff obtiene un valor de 0.28 , que se puede clasificar como pequeño. Confirmándose asíla hipótesis específica H1.5: los hombres presentan una actitud más favorable hacia la violencia contra la mujer en el factor desconsideración.

Tabla 7

Comparación de las actitudes hacia la violencia contra la mujer considerando la variable género en función al factor desconsideración

\begin{tabular}{ccccccc}
\hline Sexo & $\mathrm{n}$ & $\begin{array}{c}\text { Media de } \\
\text { Rangos }\end{array}$ & $\begin{array}{c}\text { Suma de } \\
\text { Rangos }\end{array}$ & $\begin{array}{c}\text { U de Mann } \\
\text { Whitney }\end{array}$ & $\mathrm{Z}$ & $\mathrm{d}_{\text {cliff }}$ \\
\hline Hombres & 72 & 116.13 & 8361.50 & & & \\
Mujeres & 124 & 88.26 & 10944.50 & & & \\
\hline Total & 196 & & & & & \\
\hline$* * * \mathrm{p}<.001$ & & & & & & \\
\hline
\end{tabular}

En cuanto a la tabla 8, correspondiente a la comparación de medias de rango de las actitudes hacia la violencia contra la mujer en relación de pareja entre el grupo de hombres y mujeres en función al factor roles de pareja, se observa que el primer grupo obtiene una media de rangos de 123.81 y el segundo grupo un 83.81 , con un valor $U=2642.00$ y $Z=-4.777$ ( $p<.001$ ), que indican la existencia de diferencias estadísticamente significativas entre ambos grupos. El coeficiente dcliff alcanza un valor de 0.41 que se puede clasificar como un efecto pequeño. Confirmándose, de esta forma, la hipótesis específica H1.6: los varones presentan una actitud más favorable hacia la violencia contra la mujer en el factor roles de pareja. 
Tabla 8

Comparación de las actitudes hacia la violencia contra la mujer considerando la variable género, en función al factor roles de pareja

\begin{tabular}{ccccccc}
\hline Sexo & $\mathrm{n}$ & $\begin{array}{c}\text { Media de } \\
\text { Rangos }\end{array}$ & $\begin{array}{c}\text { Suma de } \\
\text { Rangos }\end{array}$ & $\begin{array}{c}\text { U de Mann } \\
\text { Whitney }\end{array}$ & $\mathrm{Z}$ & $\mathrm{d}_{\text {cliff }}$ \\
\hline Hombres & 72 & 123.81 & 8914.00 & & & \\
Mujeres & 124 & 83.81 & 10392.00 & & & \\
\hline Total & 196 & & & & & \\
\hline$* * * \mathrm{p}<.001$ & & & & & & \\
\hline
\end{tabular}

En la comparación de las medias de rango de las actitudes hacia la violencia contra la mujer en relación de pareja entre estudiantes provenientes de instituciones educativas estatales y no estatales, usando como función al factor la violencia como medio (tabla 9), se observa que el primer grupo obtiene una media de rangos de 119.54 y el segundo grupo obtiene 73.20 , con un valor $U=2510.00$ y $Z=-5.747(p<$ .001), lo cual indica la existencia de diferencias estadísticamente significativas entre ambos grupos. El coeficiente dcliff alcanza un valor de 0.47 , que se puede clasificar como un efecto pequeño. De esta manera, se valida la hipótesis específica H2.1: los estudiantes de instituciones educativas estatales presentan una actitud más favorable hacia la violencia contra la mujer en función al factor la violencia como medio.

Tabla 9

Comparación de las actitudes hacia la violencia contra la mujer considerando el tipo de institución educativa en función al factor la violencia como medio

\begin{tabular}{ccccccc}
$\begin{array}{c}\text { Tipo de Institución } \\
\text { educativa }\end{array}$ & $\mathrm{n}$ & $\begin{array}{c}\text { Media de } \\
\text { Rangos }\end{array}$ & $\begin{array}{c}\text { Suma de } \\
\text { Rangos }\end{array}$ & $\begin{array}{c}\text { U de Mann } \\
\text { Whitney }\end{array}$ & $\mathrm{Z}$ & $\mathrm{d}_{\text {cliff }}$ \\
\hline Estatal & 107 & 119.54 & 12791.00 & & & \\
No Estatal & 89 & 73.20 & 6515.00 & & & \\
\hline Total & 196 & & & & & \\
\hline$* * * \mathrm{p}<.001$ & & & & & & \\
\hline
\end{tabular}

En la tabla 10, se presentan los resultados de la comparación de las medias de rango de las actitudes hacia la violencia contra la mujer en relación de pareja entre estudiantes provenientes de instituciones educativas estatales y no estatales, en función al factor negación a la libertad. Los resultados indican que el primer grupo obtiene una media de rangos de 116.82 y el segundo grupo 76.47 , con un valor $\mathrm{U}=2801.00$ y $Z=-4.974$ ( $p<.001$ ), lo cual indica la existencia de diferencias estadísticamente significativas entre ambos grupos. Además, el coeficiente dcliff obtiene un valor de 0.41 , que corresponde a un efecto 
pequeño. Entonces, se valida la hipótesis específica H2.2: los alumnos y alumnas de instituciones educativas estatales presentan una actitud más favorable hacia la violencia contra la mujer en función al factor negación de la libertad.

Tabla 10

Comparación de las actitudes hacia la violencia contra la mujer considerando el tipo de institución educativa en función al factor negación a la libertad

\begin{tabular}{ccccccc}
$\begin{array}{c}\text { Tipo de Institución } \\
\text { educativa }\end{array}$ & $\mathrm{n}$ & $\begin{array}{c}\text { Media de } \\
\text { Rangos }\end{array}$ & $\begin{array}{c}\text { Suma de } \\
\text { Rangos }\end{array}$ & $\begin{array}{c}\text { U de Mann } \\
\text { Whitney }\end{array}$ & $\mathrm{Z}$ & $\mathrm{d}_{\text {cliff }}$ \\
\hline Estatal & 107 & 116.82 & 12500.00 & & & \\
No Estatal & 89 & 76.47 & 6806.00 & & & \\
\hline Total & 196 & & & & & \\
\hline
\end{tabular}

$* * * p<.001$

En cuanto a la comparación de medias de rango de las actitudes hacia la violencia contra la mujer en relación de pareja, entre los estudiantes que provienen de instituciones educativas estatales y no estatales y en función al factor imposición de autoridad (tabla 11), se observa que el primer grupo obtiene una media de rangos de 120.80 y el segundo grupo 71.69 , con un valor $U=2375.00$ y $Z=-6.075(p<.001)$. Ello indica la existencia de diferencias estadísticamente significativas entre ambos grupos. El coeficiente dcliff es de 0.50 , que se puede clasificar como un efecto mediano. Así, se confirma la hipótesis específica H2.3: los estudiantes de instituciones educativas estatales presentan una actitud más favorable hacia la violencia contra la mujer en función al factor imposición de autoridad.

Tabla 11

Comparación de las actitudes hacia la violencia contra la mujer considerando el tipo de institución educativa en función al factor imposición de autoridad

\begin{tabular}{ccccccc}
\hline $\begin{array}{c}\text { Tipo de Institución } \\
\text { educativa }\end{array}$ & $\mathrm{n}$ & $\begin{array}{c}\text { Media de } \\
\text { Rangos }\end{array}$ & $\begin{array}{c}\text { Suma de } \\
\text { Rangos }\end{array}$ & $\begin{array}{c}\text { U de Mann } \\
\text { Whitney }\end{array}$ & $\mathrm{Z}$ & $\mathrm{d}_{\text {cliff }}$ \\
\hline Estatal & 107 & 120.80 & 12926.00 & & & \\
No Estatal & 89 & 71.69 & 6380.00 & & & \\
\hline Total & 196 & & & & & \\
\hline$* * * \mathrm{p}<.001$ & & & & & & \\
\hline
\end{tabular}


En la tabla 12, correspondiente a la comparación de medias de rango de las actitudes hacia la violencia contra la mujer en relación de pareja, entre los estudiantes que provienen de instituciones educativas estatales y no estatales y en función al factor machismo, los resultados muestran que el primer grupo obtiene una media de rangos de 118.37 y el segundo grupo 74.61 , con un valor $U=2635.00$ y $Z=-5.397$ ( $p$ $<$.001). Ello señala que existen diferencias estadísticamente significativas entre ambos grupos. El coeficiente dcliff alcanza un valor de 0.45 , correspondiente a un efecto pequeño. Validándose, así, la hipótesis específica H2.4: los alumnos y alumnas de instituciones educativas estatales presentan una actitud más favorable hacia la violencia contra la mujer en función al factor machismo.

Tabla 12

Comparación de las actitudes hacia la violencia contra la mujer considerando el tipo de institución educativa en función al factor machismo

\begin{tabular}{ccccccc}
\hline $\begin{array}{c}\text { Tipo de Institución } \\
\text { educativa }\end{array}$ & $\mathrm{n}$ & $\begin{array}{c}\text { Media de } \\
\text { Rangos }\end{array}$ & $\begin{array}{c}\text { Suma de } \\
\text { Rangos }\end{array}$ & $\begin{array}{c}\text { U de Mann } \\
\text { Whitney }\end{array}$ & $\mathrm{Z}$ & $\mathrm{d}_{\text {cliff }}$ \\
\hline Estatal & 107 & 118.37 & 12666.00 & & & \\
No Estatal & 89 & 74.61 & 6640.00 & & & \\
\hline Total & 196 & & & & & \\
\hline
\end{tabular}

$* * * p<.001$

Los resultados de la comparación de medias de rango de las actitudes hacia la violencia contra la mujer en relación de pareja, entre los estudiantes que provienen de instituciones educativas estatales y no estatales y en función al factor desconsideración se presentan en la tabla 13. Se observa que el primer grupo obtiene una media de rangos de 115.64 y el segundo grupo 77.89 , con un valor U $=2927.000$ y Z = 4.670 ( $\mathrm{p}<.001$ ), lo cual indica la existencia de diferencias estadísticamente significativas entre ambos grupos. El coeficiente dcliff obtiene un valor de 0.39 , que se puede clasificar como un efecto pequeño. Así, se valida la hipótesis específica $\mathrm{H2.5}$ : los estudiantes de instituciones educativas estatales presentan una actitud más favorable hacia la violencia contra la mujer en función al factor desconsideración. 
Tabla 13

Comparación de las actitudes hacia la violencia contra la mujer considerando el tipo de institución educativa, en función al factor desconsideración

\begin{tabular}{ccccccc}
\hline $\begin{array}{c}\text { Tipo de Institución } \\
\text { educativa }\end{array}$ & $\mathrm{n}$ & $\begin{array}{c}\text { Media de } \\
\text { Rangos }\end{array}$ & $\begin{array}{c}\text { Suma de } \\
\text { Rangos }\end{array}$ & $\begin{array}{c}\text { U de Mann } \\
\text { Whitney }\end{array}$ & $\mathrm{Z}$ & $\mathrm{d}_{\text {cliff }}$ \\
\hline Estatal & 107 & 115.64 & 12374.00 & & & \\
No Estatal & 89 & 77.89 & 6932.00 & & & \\
\hline Total & 196 & & & & & \\
\hline$* * * \mathrm{p}<.001$ & & & & & & \\
\hline
\end{tabular}

En la tabla 14, correspondiente a la comparación de medias de rango de las actitudes hacia la violencia contra la mujer en relación de pareja, entre los estudiantes que provienen de instituciones educativas estatales y no estatales y en función al factor roles de pareja, se observa que el primer grupo obtiene una media de rangos de 118.17 y el segundo grupo 74.85 , con un valor $U=2656.50$ y $Z=-5.344(p$ < .001). Así, se confirma la existencia de diferencias estadísticamente significativas entre ambos grupos. El coeficiente dcliff es de 0.44 , que corresponde a un efecto pequeño. Confirmándose, así, la hipótesis específica $\mathrm{H} 2.6$ : los alumnos y alumnas de instituciones educativas estatales presentan una actitud más favorable hacia la violencia contra la mujer en función al factor roles de pareja.

Tabla 14

Comparación de las actitudes hacia la violencia contra la mujer considerando el tipo de institución educativa, en función al factor roles de pareja

\begin{tabular}{ccccccc}
\hline $\begin{array}{c}\text { Tipo de Institución } \\
\text { educativa }\end{array}$ & $\mathrm{n}$ & $\begin{array}{c}\text { Media de } \\
\text { Rangos }\end{array}$ & $\begin{array}{c}\text { Suma de } \\
\text { Rangos }\end{array}$ & $\begin{array}{c}\text { U de Mann } \\
\text { Whitney }\end{array}$ & $\mathrm{Z}$ & $\mathrm{d}_{\text {cliff }}$ \\
\hline Estatal & 107 & 118.17 & 12644.50 & & & \\
No Estatal & 89 & 74.85 & 6661.50 & & & \\
\hline Total & 196 & & & & & \\
\hline$* * * \mathrm{p}<.001$ & & & & & & \\
\hline
\end{tabular}




\section{Discusión}

Los resultados obtenidos del análisis estadístico son expuestos y dilucidados en este capítulo, permitiendo dar una explicación más concienzuda de las actitudes hacia la violencia contra la mujer en función al género de los participantes y al tipo de gestión de la institución educativa.

Se halló en la investigación que los estudiantes varones del cuarto año de secundaria tienen una actitud más favorable hacia la violencia contra la mujer en la relación de pareja, considerando tanto cada uno de los factores de la escala (la violencia como medio, negación de la libertad, imposición de autoridad, machismo, desconsideración y roles de pareja) como el puntaje total de la escala, con resultados similares a los presentados en investigaciones precedentes como la de Chuquimaju (2000), Elizalde (2001), Rodríguez et al. (2006), Ferrer et al. (2006), Macazana (2010) y Gracia et al. (2010), tanto en estudiantes de secundaria como en universitarios y adultos; así, se puede relacionar lo encontrado con lo que afirma Corsi, Dohmen, Sotes y Bonino (1996) respecto a la posición que asume el hombre frente a la violencia contra la mujer, pues éste tiende a eludir su responsabilidad, justificando y minimizando su conducta violenta en función al comportamiento o actitudes de provocación, atribuidos a la mujer. También se puede vincular estos resultados con lo reportado por Rodríguez et al. (2006), quienes encontraron un grupo de los participantes en su investigación que consideraba el maltrato a la mujer como un problema de pareja, donde nadie debe intervenir. Asimismo, en la investigación de Gracia et al. (2010) se encontró que quienes mostraron actitudes positivas hacia la denuncia de violencia contra la mujer eran menos tolerantes con la violencia de pareja contra la mujer, percibiéndola como un problema social importante y sin culpar a las víctimas.

En el caso de las mujeres estudiantes del cuarto año de secundaria, se encontraron resultados correspondientes a una actitud de indiferencia respecto a la violencia contra la mujer en la relación de pareja, resultado similar al de Macazana (2010) en poblaciones de estudiantes universitarios; indicando, así, que aún se aprecia una pasividad en las mujeres impuesta por la sociedad, donde se mantiene su predominancia patriarcal, como lo manifiesta Velázquez (2003), al señalar que la pasividad femenina es un estereotipo que ha sido construido culturalmente, situando a las mujeres en posición de víctimas por el solo hecho de ser mujeres. La pasividad se ve feminizada porque el imaginario atribuye a las mujeres, en el contexto de la violencia, las características de ser sumisas, obedientes, propensas a ser atacadas, poca capacidad para defenderse y miedos concretos frente a la fuerza y el poder de quien la agrede.

Mediante el análisis de los hallazgos anteriores, se puede afirmar que en los participantes existen diferencias estadísticamente significativas en las actitudes hacia la violencia contra la mujer entre los estudiantes varones y mujeres de cuarto año de secundaria. Además, se corresponde al menos a una magnitud pequeña del efecto. De esta manera, este resultado confirma lo señalado por Velázquez (2003), pues este tipo de violencia es inseparable de la noción de género porque se basa y se ejerce en y por la diferencia social y subjetiva entre los sexos.

En cuanto a la comparación, considerando el tipo de gestión educativa, se encontró que los estudiantes de instituciones educativas estatales tenían una actitud más favorable hacia la violencia contra la mujer que los de las instituciones educativas no estatales (tanto al considerar cada uno de los factores como el puntaje total de la escala). Estos resultados difieren de los hallazgos de la investigación de Macazana (2010), en función al tipo de institución y en una población universitaria, pero coinciden con lo reportado en algunas investigaciones como la de Ferrer et al. (2006), que plantean que el tipo de educación recibida y los modelos observados (particularmente la educación igualitaria entre hermanos y hermanas y la situación laboral de los padres) contribuyen también a predecir el sexismo y la tolerancia hacia la violencia contra las mujeres en la pareja. Los resultados de la investigación de Gracia et al. (2010) también, sugieren la necesidad de una mejor educación dirigida a aquellos grupos que mantienen actitudes de aceptabilidad y tolerancia hacia la violencia de pareja, para lograr una prevención más eficaz. 


\section{Conclusiones}

Los alumnos varones de cuarto año de secundaria presentan actitudes más favorables hacia la violencia contra la mujer que sus pares mujeres, considerando el puntaje total de la escala, así como en los factores: Negación a la libertad, imposición de autoridad, machismo, desconsideración y roles de pareja. Por otro lado, no se encontró diferencias estadísticamente significativas en las actitudes hacia la violencia contra la mujer entre los estudiantes varones y mujeres del cuarto año de secundaria, en función al factor la violencia como medio.

Asimismo, los alumnos que provienen de instituciones educativas estatales presentan actitudes más favorables hacia la violencia contra la mujer en relación de pareja que sus pares de instituciones educativas no estatales, considerando el puntaje total de la escala, como en cada uno de los seis factores del instrumento.

\section{Referencias}

Anicama, J., Vizcardo, S., Carrasco, J., y Mayorga, E. (1999). Estudio Epidemiológico sobre la violencia y comportamientos asociados en Lima Metropolitana y Callao. MINSA.

Carmona, L. S., Doporto, B., Corral, S., Villalobos, R., y López, S. (2005). Violencia contra la mujer universitaria en las relaciones de parejas. Revista Santiago, 108, 248-255.

Chuquimajo, S. (2000). Actitudes hacia la violencia contra la mujer en la relación de pareja en estudiantes de 5 to año de secundaria de Lima Metropolitana (Tesis de pregrado). Universidad Nacional Mayor de San Marcos. Lima, Perú.

Comisión Económica para América Latina y el Caribe (2007). ¡Ni una más! El derecho a vivir una vida libre de violencia en América Latina $y$ el Caribe. Recuperado de: https://www.cepal.org/es/publicaciones/2870-ni-mas-derecho-vivir-vida-libre-violencia-americalatina-caribe

Corsi, J., Dohmen, L., Sotes, M., y Bonino, L. (1996). Violencia masculina en la pareja. Una aproximación al diagnóstico y a los modelos de intervención. Buenos aires, Argentina: Paidós.

Elizalde, R. (2001). Actitudes hacia la violencia y rasgos de personalidad en estudiantes universitarios de Lima (Tesis de Maestría). Universidad Nacional Mayor de San Marcos. Lima, Perú.

Fernández, A., y Fernández, I. M. (2009). Crítica y alternativas a la significación estadística en el contraste de hipótesis. Madrid, España: La Muralla.

Ferrer, V., Bosch, E., Ramis, M., y Navarro C. (2006). Las creencias y actitudes sobre la violencia contra las mujeres en la pareja: Determinantes sociodemográficos, familiares y formativos. Anales de psicología, 22(2), 251-259.

Gracia, E., Herrero, J., Lila, M., y Fuente, A. (2010). Percepciones y Actitudes hacia la Violencia de Pareja contra la Mujer en Inmigrantes Latinoamericanos en España. Psychosocial Intervention, 19(2), 135144.

Grisson, R. J., y Kim, J. J. (2012). Effect sizes for research: Univariate and multivariate applications. (2nd ed.) New York, NY: Taylor \& Francis.

Güezmes, A., Palomino, N., y Ramos, M. (2002). Violencia sexual y física contra las mujeres en el Perú. Estudio multicéntrico de la OMS sobre la violencia de pareja y la salud de las mujeres.

Horno, P. (2009). Amor y violencia. La dimensión afectiva del maltrato. Madrid, España: Editorial Desclée de Brouwer, S.A.

Lorente, M. (2008). Violencia y maltrato de género: Aspectos generales desde la perspectiva sanitaria. Emergencias: Revista de la Sociedad Española de Medicina de Urgencias y Emergencias, 20(3), 191197. Recuperado de: https://dialnet.unirioja.es/servlet/articulo?codigo $=2665314$

Macazana, J. (2010). Actitudes hacia la violencia contra la mujer en la relación de pareja en estudiantes universitarios de cuatro universidades de Lima Metropolitana considerando el género, nivel 
socioeconómico y el que hayan tenido o no una pareja (Tesis de pregrado). Universidad Nacional Mayor de San Marcos. Lima, Perú.

Matud, P., Rodríguez C., Marrero, R., y Carballeira, M. (2002). Psicología del género: Implicaciones en la vida cotidiana. Madrid, España: Editorial Biblioteca Nueva, S. I.

OMS (2002). Informe mundial sobre la violencia y la salud. Recuperado de: https://www.who.int/violence_injury_prevention/violence/world_report/es/summary_es.pdf

Ons, S. (2009). Violencia/s. Buenos Aires, Argentia: Editorial Paidós.

Ortega, J. (2011). Intervención contra la violencia de género para las tutorías de la ESO. Madrid, España: Pirámide.

Solano, P., y Velzeboer, M. (2003). Componentes clave en la formulación de leyes y políticas contra la violencia hacia las mujeres. Recuperado de: http://grupocisalva.univalle.edu.co/bpr2/esp/Descargas/publicaciones/Componentes_clave_en_f ormulacion_leyespoliticas_contraviolenciademujeres.pdf

Rocha, T. (2011). Idēntidades de género, más allá del cuerpo y mitos. México: Trillas.

Rodríguez, V., Sánchez, C. y Alonso, D. (2006). Creencias de adolescentes y jóvenes en torno a la violencia de género y las relaciones de pareja. Portularia, VI (2), 189-204.

Ruiz, P. (2006) El maltrato a la mujer. Enfoque psicoanalítico a través de su historia y su clínica. Madrid, España: Editorial Síntesis.

Velázquez, S. (2003). Violencias cotidianas, violencias de género. Buenos Aires, Argentina: Paidós. 